\title{
Research Progresses on the Relationship Between the Phthalates Exposure and Children's Neurodevelopmental Disorders
}

\author{
Yu Qifan ${ }^{1}$, Shen Xuezhi ${ }^{1}$, Chen Hongqing ${ }^{1}$, Chen Qianye ${ }^{1}$, Zheng Guofen ${ }^{1}$, Luo Yufeng ${ }^{1}$, Li Aiqing ${ }^{2}$, \\ Ding Yuemin ${ }^{1, *}$ \\ ${ }^{1}$ Department of Clinical Medicine, School of Medicine, Zhejiang University City College, Hangzhou, China \\ ${ }^{2}$ Gastroenterology Laboratory, Sir Run Run Shaw Hospital, Zhejiang University School of Medicine, Hangzhou, China
}

Email address:

dingyuemin@zucc.edu.cn (Ding Yuemin)

*Corresponding author

\section{To cite this article:}

Yu Qifan, Shen Xuezhi, Chen Hongqing, Chen Qianye, Zheng Guofen, Luo Yufeng, Li Aiqing, Ding Yuemin. Research Progresses on the Relationship Between the Phthalates Exposure and Children's Neurodevelopmental Disorders. Science Journal of Public Health.

Vol. 9, No. 3, 2021, pp. 72-76. doi: 10.11648/j.sjph.20210903.11

Received: March 6, 2021; Accepted: April 26, 2021; Published: May 8, 2021

\begin{abstract}
Neurodevelopment, a complex multifactorial process, is influenced by genetic factors, environmental factors and the interaction between them. Among various environmental factors, prenatal or postnatal phthalate exposure are gradually attracting great attention. The plasticizers phthalate are widely used in various products. Since they do not form covalent bonds with the polymer matrix, they may leach or outgas into the environment and enter the human body, even affect the development of the fetus or newborn through the placenta or breast milk. In recent years, increasing evidences have shown that maternal phthalate exposure can interfere with the development of fetal central nervous system, resulting in neurodevelopmental disorders in postnatal children. However, the specific relationship between maternal phthalate exposure and fetal neurodevelopment and its mechanism have not been clearly studied. At present, the known possible mechanisms include disruption of thyroid homeostasis, alteration of calcium signal transduction, disturbance of lipid metabolism, interfering with the action of sex hormones, influence of the Rho-GTPase pathway, induction of epigenetic and oxidative stress, etc. As the problem has gradually become the focus of attention all over the world, new research results are still emerging. In order to provide references for further study of the etiology and pathogenesis, the latest research progresses and mechanisms of phthalate exposure on neurodevelopment were reviewed in the following article.
\end{abstract}

Keywords: Phthalates, Plasticizer, Neurodevelopment, Mechanism

\section{Overview of Phthalates}

Phthalates are a group of common plasticizers which are widely used in various daily necessities, such as plastic containers, medical equipment and food packaging [1]. According to their molecular weight and chemical properties, phthalates are usually divided into two types of different application and toxicological properties [2]. The high molecular weight phthalates $(M W \geq 50 \mathrm{Da})$, such as di (2-ethylhexyl) phthalate (DEHP), are often added to products to enhance their flexibility and strength. The low molecular weight (LMW) phthalates ( $\mathrm{MW}<250 \mathrm{Da}$ ), such as di-n-butyl phthalate (DnBP) and diisobutyl phthalate (DiBP), are commonly used in personal care products, medical supplies, cosmetics and packaging materials $[2,3]$. Because these compounds do not form covalently bound to the polymer matrix, they may be released and migrate into the surrounding environment [4]. The human body may be exposed to them by skin contact, ingestion, inhalation and also transfer from the mother to the fetus or newborn through placenta or breastfeeding [5]. Phthalates have a short biological half-life, ranging from several hours to several days, and their metabolites are detected in human blood, urine, feces, breast milk and amniotic fluid $[6,7]$.

The concentration of phthalate metabolites in urine is currently used as an evaluation index of exposure [8]. In 
general, the level of phthalate metabolites in urine of children is higher than that of adults, while the level of phthalate metabolites in urine of women is significantly higher than that of men [6]. Because of its wide range of application and easy accesses into the human body, the biological impact of phthalates and their metabolites has gradually become a hot issue for researchers and the public. In this review, we describe recent research progress on the mechanisms of phthalates' influence on neurodevelopment that provide the basis of prevention.

\section{Environmental Factors and Neurodevelopmental Disorders in Children}

Neurodevelopmental disorders are a group of diseases characterized by social delay or mental retardation, including specific language delay, autism spectrum disorders (ASD), attention deficit hyperactivity disorder (ADHD), learning disabilities and motor delays. In the early stage of development, human brain is sensitive to environmental neurotoxins. Epidemiological studies have found that exposure to environmental toxins during embryo stage causes neurodevelopmental disorders, particularly when the pregnant women smoke or persistently exposed to plastic-derived toxins (such as phthalates). In addition, there is evidence that environmental toxins cause epigenetic changes, such as DNA methylation [9]. If the prenatal brain during a period of rapid growth is exposed to environmental toxins, especially the endocrine disruptor chemicals, it may result in neurodevelopmental disorders related diseases such as ASD and ADHD [10].

\section{Effects of Phthalates on Neurodevelopment}

Phthalates and their metabolites in the environment affect the neurodevelopment of fetuses and infants [11]. Studies have reported that prenatal exposure to phthalates associated with children's neurobehavioral difficulties [12]. A cohort study in Taiwan showed that higher concentrations of DBP and DEHP in mother's urine samples were associated with children's development of conduct problems and ADHD related symptoms [13]. In addition, there is a positive correlation between the concentration of DEHP metabolites in urine and the symptoms of ADHD [14]. Choi and colleagues evaluated the relationship between prenatal phthalate exposure and comprehensive assessment of executive function (EF) in 262 preschool children with clinically significant/subthreshold ADHD symptoms and 78 typically developing children, and found that exposure to mono-benzyl phthalate $(\mathrm{MBzP})$ during pregnancy was associated with poorer EF in both sexes [12].

Testa and colleagues evaluated the level of the primary and secondary metabolites of DEHP in 48 ASD children and 45 healthy controls. Compared with the control group, the urinary 5-OH-MEHP and 5-oxo-MEHP concentration significantly increased in children with autism. In addition, the urinary 5-oxo-MEHP (fully oxidized form) showed $91.1 \%$ specificity in identifying children with ASD [15]. These findings suggested for the first time an association between phthalate exposure and ASD.

\section{Possible Mechanisms of Phthalate Affecting Neurodevelopment}

\subsection{Disruption of Thyroid Homeostasis}

Thyroid hormones are essential for the neural development of prenatal fetus and postpartum newborn. The maturation of the brain, such as the migration of granular and pyramidal cells in the cortex, are regulated by thyroid hormones [14]. Epidemiological studies have found that early life phthalate exposure may influence thyroid function in school-age children. Villanger and colleagues examined the relationships between concentrations of 12 urinary phthalate metabolites and 6 plasma thyroid function biomarkers among 1072 Norwegian pregnant women, and found that mono-butyl phthalate (MBP) was associated with increased total triiodothyronine (TT3) and free T3 index (fT3i), while DEHP was associated with a decrease in TT3 and fT3i [16].

\subsection{Alteration of Intracellular Calcium Signal Transduction}

Intracellular calcium plays an important role in neurotransmitter releases, excitability regulation and gene expression of nerve cells [10]. In the nerve terminals of rat pituitary glands and pheochromocytoma cells, low dose $(800 \mathrm{nM})$ of DEHP increases the intracellular calcium level [17]. In the testis of zebrafish Danio rerio, acute exposure to bis (2-ethylhexyl) phthalate (BEHP) disrupts testicular $\mathrm{Ca}^{2+}$ homeostasis by increasing $\mathrm{Ca}^{2+}$ influx [18]. In human cell lines, BBP and DBP metabolites interfere with calcium signal transduction coupled with nicotinic acetylcholine receptors (nAChRs) [10, 19].

\subsection{Disturbance of Lipid Metabolism}

DEHP may inhibit myelination by affecting lipid metabolism and distribution [20]. Development of normal fetal brain depends on appropriate levels of lipids and their fatty acid substituents, including docosahexaenoic acid (DHA) and arachidonic acid (AA) [10, 21]. Maternal exposure to DEHP from day 0 to day 19 of pregnancy reduced the concentration of essential lipids that synthesize DHA and AA [20]. In liver and hepatocytes, DBP exposure could induce liver lipid metabolism disorder and other hepatic toxicity through the peroxisome proliferator activated receptor (PPAR $\alpha$ ) signal pathway [22]. Similarly, the effects of DEHP on fetal lipid metabolism also are mediated by PPAR activity. 


\subsection{Androgen Effect of DEHP}

The antiandrogenic effects of several phthalates such as DEHP, BBP, DnBP and DiBP may affect neurodevelopment. Both animal and epidemiological studies have found that phthalate exposure interfere with homeostasis of the sex hormones (progesterone, androstenedione and testosterone) in vivo [7, 23]. Phthalates interfere with aromatase activity. Aromatase is an enzyme that plays an important role in reproduction and neuroprotection. When rats were exposed to LMW phthalates such as DBP, aromatase in hippocampus was up-regulated and estrogen receptor $\beta$ was down regulated, thus reducing the expression of cAMP response element binding protein (CREB) and brain derived neurotrophic factor (BDNF) [24]. Although evidence of some cases is weak or indirect, the importance of phthalates' anti-androgen effects on brain development cannot be ignored [25].

\subsection{Dysregulation of the Neurotransmitter System}

In mammalian brains, the inhibitory transmitter gamma-aminobutyric acid (GABA) plays an extremely significant role in critical neurodevelopmental stages. The balance between GABA mediated inhibition and L-glutamate (Glu) mediated excitation is of crucial importance in the brain by affecting connectivity and synapse pruning [26]. It has been reported that postnatal exposure to DEHP significantly reduces GABA concentration in the hypothalamus [27]. Dopamine is another neurotransmitter which closely related to cognition, emotion, learning and memory. Studies have found that the expression of dopamine receptor D2 was decreased in in striatum of the female mice after pubertal exposure to DEHP, which might be associated with the DEHP-induced changes of social behavior [28].

\subsection{Influence of the Rho-GTPase Pathway}

Chronic exposure to DBP in vivo leads to the impairment of spatial learning and memory in adult male mice, while acute incubation of DBP in vitro inhibits neurite growth and migration in neuron cells. DBP exposure inhibits Rac1/PAK1/LIMK1 activity in the Rho-GTPase pathway of the small $\mathrm{G}$ protein family and activate RhoA/ROCK/LIMK2 signal transduction simultaneously. Finally, it stimulated the phosphorylation of cofilin and inhibited the cofilin-mediated turnover of F-actin, thus severely inhibiting the growth of neurites [29].

\subsection{Other Aspects}

DEHP and DBP have epigenetic toxicity. Maternal exposure to DEHP increases DNA methylation and the expression levels of DNA methyltransferase in the testes of mice, and the epigenetic changes may also affect behavior [30].

DEHP is metabolized to mono-2-ethylhexyl phthalate (MEHP). Therefore, the effect of MEHP may represent the toxic effect of DEHP. The rat pheochromocytoma (PC12) cells are used as neuronal differentiation model due to their responses to nerve growth factor (NGF). Choline acetyltransferase (ChAT) and tyrosine hydroxylase $(\mathrm{TH})$ are markers of cholinergic neurons and dopaminergic neurons, respectively. MEHP can up-regulate ChAT mRNA level, while down-regulate TH mRNA level, indicating that MEHP exposure regulates neuronal differentiation [31]. The results suggest the effects of phthalates, especially MEHP on midbrain dopaminergic neurons, the later are known to associated with ADHD or schizophrenia [32].

Oxidative stress is one of the major causes of neuronal damage, which might be a chemically induced process. Ponsonby and colleagues investigated the interplay between maternal prenatal phthalate levels, infant genetic vulnerability to oxidative stress, and child neurodevelopment, and found that prenatal phthalate levels and infant oxidative stress-related genetic vulnerability are associated with adverse neurodevelopment [33]. Consistently, animal experiment showed that neurobehavioral changes induced by DBP may be mediated by oxidative damage in the mouse brain [34].

\section{Summary and Outlook}

To sum up, the possible mechanisms of phthalate esters on neurodevelopment include disruption of thyroid homeostasis, alteration of calcium signal transduction, disturbance of lipid metabolism, interfering with the action of sex hormones, influence of the Rho-GTPase pathway, induction of epigenetic and oxidative stress, etc.

In addition, epidemiological and laboratory studies on the relationship between phthalate exposure and neurodevelopmental disorders in children are not completely consistent. The potential reasons for heterogeneity are the following inconsistencies in the experimental design: sample size; time point and frequency of measurement; phthalate metabolites; neurodevelopmental testing methods and age of children at the time of evaluation. This requires future researchers to be more complete and comprehensive in experimental designs.

Phthalates are ubiquitous in our daily life. Although people have gradually paid attention to the various health hazards that they may cause, the exposure to phthalates seems unavoidable at present. Therefore, it is still an urgent problem to fully understand the relationship between phthalates and neurodevelopmental disorders. Further studies of the toxicological mechanisms will help us to find out the cause of neurodevelopmental disorders and propose corresponding preventive measures.

\section{Acknowledgements}

This work was supported by the Basic public welfare research project of Zhejiang Province (No. LGD19H090008), the Natural Science Foundation of Zhejiang Province (No. LY19H260007) and the Zhejiang Medical and Health Science and Technology Program (No. 2019KY412). 


\section{References}

[1] Giuliani A, Zuccarini M, Cichelli A, et al. (2020). Critical review on the presence of phthalates in food and evidence of their biological impact [J]. Int J Environ Res Public Health, 17 (16): 5655 .

[2] Qian X, Li J, Xu S, et al. (2019). Prenatal exposure to phthalates and neurocognitive development in children at two years of age [J]. Environ Int, 131: 105023.

[3] Schettler T. (2006). Human exposure to phthalates via consumer products [J]. Int J Androl, 29 (1): 134-139.

[4] Yost EE, Euling SY, Weaver JA, et al. (2019). Hazards of diisobutyl phthalate (DIBP) exposure: A systematic review of animal toxicology studies [J]. Environ Int, 125: 579-594.

[5] Xia Y. (2020). Early-life environmental exposure and disease: Springer, Singapore.

[6] Katsikantami I, Sifakis S, Tzatzarakis MN, et al. (2016). A global assessment of phthalates burden and related links to health effects [J]. Environ Int, 97: 212-236.

[7] Zhang Q, Chen XZ, Huang X, et al. (2019). The association between prenatal exposure to phthalates and cognition and neurobehavior of children-evidence from birth cohorts $[\mathrm{J}]$. Neurotoxicology, 73: 199-212.

[8] Haug LS, Sakhi AK, Cequier E, et al. (2018). In-utero and childhood chemical exposome in six European mother-child cohorts [J]. Environ Int, 121 (Pt 1): 751-763.

[9] Tran NQV, Miyake K. (2017). Neurodevelopmental Disorders and Environmental Toxicants: Epigenetics as an Underlying Mechanism [J]. Int J Genomics, 2017: 7526592.

[10] Miodovnik A, Edwards A, Bellinger DC, et al. (2014). Developmental neurotoxicity of ortho-phthalate diesters: review of human and experimental evidence [J]. Neurotoxicology, 41: 112-122.

[11] Radke EG, Braun JM, Nachman RM, et al. (2020). Phthalate exposure and neurodevelopment: A systematic review and meta-analysis of human epidemiological evidence [J]. Environ Int, 137: 105408 .

[12] Choi G, Villanger GD, Drover SSM, et al. (2021). Prenatal phthalate exposures and executive function in preschool children [J]. Environ Int, 149: 106403.

[13] Lien YJ, Ku HY, Su PH, et al. (2015). Prenatal exposure to phthalate esters and behavioral syndromes in children at 8 years of age: Taiwan Maternal and Infant Cohort Study [J]. Environ Health Perspect, 123 (1): 95-100.

[14] Chopra V, Harley K, Lahiff M, et al. (2014). Association between phthalates and attention deficit disorder and learning disability in U.S. children, 6-15 years [J]. Environ Res, 128: 64-69.

[15] Testa C, Nuti F, Hayek J, et al. (2012). Di- (2-ethylhexyl) phthalate and autism spectrum disorders [J]. ASN Neuro, 4 (4): 223-229.

[16] Villanger GD, Drover SSM, Nethery RC, et al. (2020). Associations between urine phthalate metabolites and thyroid function in pregnant women and the influence of iodine status [J]. Environ Int, 137: 105509.
[17] Tully K, Kupfer D, Dopico AM, et al. (2000). A plasticizer released from IV drip chambers elevates calcium levels in neurosecretory terminals [J]. Toxicol Appl Pharmacol, 168 (3): 183-188.

[18] Batista-Silva H, Dambros BF, Rodrigues K, et al. (2020). Acute exposure to bis (2-ethylhexyl)phthalate disrupts calcium homeostasis, energy metabolism and induces oxidative stress in the testis of Danio rerio [J]. Biochimie, 175: 23-33.

[19] Liu PS, Tseng FW, Liu JH. (2009). Comparative suppression of phthalate monoesters and phthalate diesters on calcium signalling coupled to nicotinic acetylcholine receptors $[\mathrm{J}]$. J Toxicol Sci, 34 (3): 255-263.

[20] Xu Y, Agrawal S, Cook TJ, et al. (2007). Di(2-ethylhexyl)-phthalate affects lipid profiling in fetal rat brain upon maternal exposure [J]. Arch Toxicol, 81 (1): 57-62.

[21] Harauma A, Hatanaka E, Yasuda H, et al. (2017). Effects of arachidonic acid, eicosapentaenoic acid and docosahexaenoic acid on brain development using artificial rearing of delta-6-desaturase knockout mice [J]. Prostaglandins Leukot Essent Fatty Acids, 127: 32-39.

[22] Zhang W, Li JY, Wei XC, et al. (2021). Effects of dibutyl phthalate on lipid metabolism in liver and hepatocytes based on PPARalpha/SREBP-1c/FAS/GPAT/AMPK signal pathway [J]. Food Chem Toxicol, 149: 112029.

[23] Hannon PR, Brannick KE, Wang W, et al. (2015). Di (2-ethylhexyl) phthalate inhibits antral follicle growth, induces atresia, and inhibits steroid hormone production in cultured mouse antral follicles [J]. Toxicol Appl Pharmacol, 284 (1): 42-53.

[24] Li X, Fang EF, Scheibye-Knudsen M, et al. (2014). Di(2-ethylhexyl) phthalate inhibits DNA replication leading to hyperPARylation, SIRT1 attenuation, and mitochondrial dysfunction in the testis [J]. Sci Rep, 4: 6434.

[25] Gore AC, Martien KM, Gagnidze K, et al. (2014). Implications of prenatal steroid perturbations for neurodevelopment, behavior, and autism [J]. Endocr Rev, 35 (6): 961-991.

[26] Howard MA, Rubenstein JL, Baraban SC. (2014). Bidirectional homeostatic plasticity induced by interneuron cell death and transplantation in vivo [J]. Proc Natl Acad Sci U S A, 111 (1): 492-497.

[27] Carbone S, Ponzo OJ, Gobetto N, et al. (2019). Effect of di (2-ethylhexyl) phthalate on the neuroendocrine regulation of reproduction in adult male rats and its relationship to anxiogenic behavior: Participation of GABAergic system [J]. Hum Exp Toxicol, 38 (1): 25-35.

[28] Wang R, Xu X, Zhu Q. (2016). Pubertal exposure to di(2-ethylhexyl) phthalate influences social behavior and dopamine receptor D2 of adult female mice [J]. Chemosphere, 144: 1771-1779.

[29] Ding Y, Lu L, Xuan C, et al. (2017). Di-n-butyl phthalate exposure negatively influences structural and functional neuroplasticity via Rho-GTPase signaling pathways [J]. Food Chem Toxicol, 105: 34-43.

[30] Tops S, Habel U, Radke S. (2019). Genetic and epigenetic regulatory mechanisms of the oxytocin receptor gene (OXTR) and the (clinical) implications for social behavior [J]. Horm Behav, 108: 84-93. 
[31] Chen T, Yang W, Li Y, et al. (2011). Mono- (2-ethylhexyl) phthalate impairs neurodevelopment: inhibition of proliferation and promotion of differentiation in PC12 cells [J]. Toxicol Lett, 201 (1): 34-41.

[32] Aumann TD. (2016). Environment- and activity-dependent dopamine neurotransmitter plasticity in the adult substantia nigra [J]. J Chem Neuroanat, 73: 21-32.
[33] Ponsonby AL, Symeonides C, Saffery R, et al. (2020). Prenatal phthalate exposure, oxidative stress-related genetic vulnerability and early life neurodevelopment: A birth cohort study [J]. Neurotoxicology, 80: 20-28.

[34] Yan B, Guo J, Liu X, et al. (2016). Oxidative stress mediates dibutyl phthalateinduced anxiety-like behavior in Kunming mice [J]. Environ Toxicol Pharmacol, 45: 45-51. 